\title{
Transition from regular motion to chaos in a logarithmic potential
}

\author{
G. I. Karanis and N. D. Caranicolas
}

Department of Physics, Section of Astrophysics, Astronomy and Mechanics, University of Thessaloniki, 54006 Thessaloniki, Greece

Received 12 October 2000 / Accepted 1 December 2000

\begin{abstract}
We investigate the properties of motion in a logarithmic galactic potential. The model can be considered to describe the motion in the meridian, $r-z$ plane, of an elliptical galaxy with a dense nucleus or bulge of radius $c$. For a given value of $c$, there is a critical value of the angular momentum $L_{z c}$ such as for $L_{z} \leq L_{z c}$, stars, moving near the galactic plane, are scattered to higher scale $z$ heights displaying chaotic motion. Our numerical calculations show that there exists a linear relationship between the radius of the nucleus and the critical value of the angular momentum. This linear relationship can be found using some elementary theoretical arguments. We use the distribution of radial velocities in order to distinguish ordered from chaotic motion. Comparison with previous work is also made.
\end{abstract}

Key words. galaxies: kinematics and dynamics

\section{Introduction}

The study of chaotic motion in celestial mechanics and galactic dynamics has been an active field of research during the last decades (see Lemaitre 1984; Innanen 1985; Henrard 1988; Caranicolas 1990; Henrard \& Caranicolas 1990; Caranicolas \& Karanis 1999). The chaotic behavior of a system can be detected using the classic method of the Poincare surface of section. One can also use, among others, the Lyapunov characteristic exponents or the dynamical spectra, based on the distribution of the values of various parameters along an orbit (Contopoulos et al. 1995; Caranicolas \& Vozikis 1999).

Of special interest is to study the transition from regular to chaotic motion as the initial conditions, the parameters or some physical quantities of a dynamical system change. Such studies, of chaotic orbits in galactic mass models with high central concentrations, have been made by Caranicolas \& Innanen (1991) and Caranicolas (1997). In the above two cases it was found that stars, with values of angular momentum equal or smaller than a critical value $L_{z c}$, were scattered to the halo upon encountering the nuclear region displaying chaotic motion.

Send offprint requests to: N. D. Caranicolas, e-mail: caranic@helios.astro.auth.gr
In the present paper we shall study the transition from regular to chaotic motion in the logarithmic potential

$V(r, z)=\frac{1}{2} \ln \left(r^{2}+\alpha z^{2}+c^{2}\right)$

where $r, z$ are the usual cylindrical coordinates while $\alpha$, $c$ are parameters. This potential is important for galactic dynamics and represents an elliptical galaxy, with a nucleus or bulge of radius $c$, which displays a flat rotation curve at large radii (see Binney \& Tremaine 1987). The parameter $1 \leq \alpha \leq 2$ defines the axial ratio of the equipotential ellipsoids. Logarithmic potentials have been frequently used by many investigators, the last two decades in order to model galactic motion. (see e.g. Richstone 1980, 1982; Caranicolas \& Vozikis 1986; Contopoulos \& Seimenis 1990; Papaphilippou \& Laskar 1996 and references therein).

The aim of the present work is to present numerical evidence that low angular momentum stars moving near the galactic plane, when approaching the nucleus, are scattered to higher scale $z$ heights, displaying chaotic motion. We shall use numerical experiments to support the idea, that low angular momentum stars, in the model (1), representing an elliptical galaxy with a nucleus or bulge, when approaching the nuclear region, show similar behavior to that found in disk galaxy models (see Caranicolas \& Innanen 1991). Furthermore, we shall find the relationship between the critical value of the angular momentum 
and the radius of the nucleus and try to explain it using numerical evidence together with some theoretical arguments. The transition from regular to chaotic motion is studied in Sect. 2. In Sect. 3 we use the distribution of radial velocities to distinguish between regular and chaotic orbits in the potential (1). Finally, in Sect. 4, we present a discussion and the conclusion of this work.

\section{Transition from regular to chaotic motion}

The Hamiltonian to the potential (1) is

$H=\frac{1}{2}\left(p_{r}^{2}+p_{z}^{2}+\frac{L_{z}^{2}}{r^{2}}\right)+\frac{1}{2} \ln \left(r^{2}+\alpha z^{2}+c^{2}\right)=h$,

where $p_{r}, p_{z}$ are the momenta per unit mass conjugate to $r$ and $z$ respectively, $L_{z}$ is the numerical value of the angular momentum while $h$ is the numerical value of $H$. Orbits were started near $r=r_{\max }$ with $p_{r}=z=0$ while the value of $p_{z}$ was found using the energy integral (2). The value $r_{\max }$ is the maximal root of the equation

$\frac{L_{z}^{2}}{2 r^{2}}+\frac{1}{2} \ln \left(r^{2}+c^{2}\right)=h$,

which is solved numerically. Figure 1 shows the relationship between the critical value of the angular momentum and the radius of the nucleus or bulge. Here one can see a straight line. Orbits with values of $L_{z c}$ and $c$ on the left part of the plane, including the line, are chaotic while orbits with the values of the parameters on the right part are regular.

Figures 2a,b show the $r-p_{r} \quad\left(z=0, p_{z}>0\right)$ surface of section and the corresponding trajectory for an orbit with initial conditions $r=0.8992, z=p_{r}=0$ while the value of $p_{z}$ is found from the energy integral. The values of the parameters are $c=0.01, h=-0.1$ while the value of angular momentum is $L_{z}=0.10$. As one can see the orbit is chaotic. Figures $2 \mathrm{c}, \mathrm{d}$ are similar to Figs. 2a,b but for $L_{z}=0.35$. Initial conditions $r=0.8273$, $z=p_{r}=0$ and the value of $p_{z}$ is found using the energy integral. Here the motion is regular. It is of interest to note that regular orbits stay near the galactic plane while chaotic orbits approach high values of $z$. Thus one can say that the behavior observed in disk galaxies (Caranicolas \& Innanen 1991) is also observed in elliptical galaxies using a quite different galactic model. This situation supports the idea that low angular momentum stars display chaotic motion in disk and elliptical galaxies with high central condensations.

The linear relationship between $c$ and $L_{z c}$ can be found semi-analytically. In fact we use essentially similar arguments to those used in Caranicolas \& Innanen (1991). On approaching the nucleus there is a change in the star's angular momentum in the $z$ direction given by

$M \Delta p_{z}=<F_{z}>\Delta t$

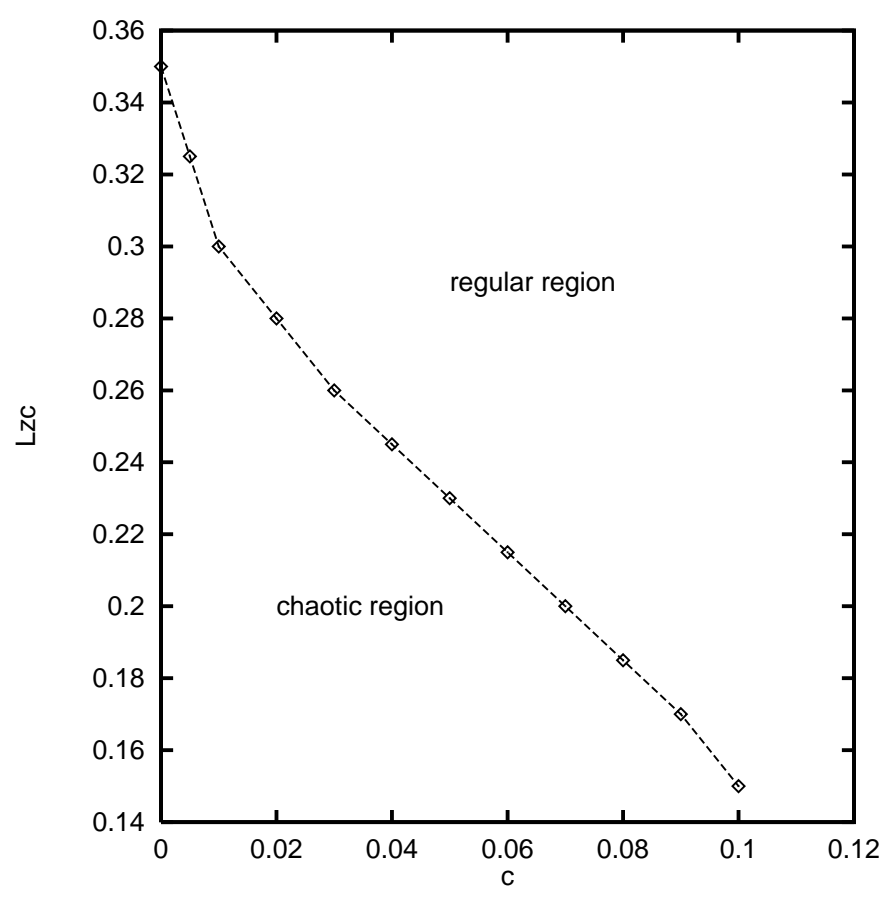

Fig. 1. Relationship between the critical value of the angular momentum $L_{z c}$ and the radius $c$. Note that the slope changes for very small values of $c$

where $M$ is the mass of the star $\left\langle F_{z}\right\rangle$ is the total average force acting in the $z$ direction while $\Delta t$ is the duration of the encounter. Empirical evidence shows that star's rise proceeds in each case cumulatively, a little more with each successive pass by the nucleus, rather than with a single "violent" encounter. We assume that a star gains considerable height after $n(n>1)$ encounters, when the total change in the momentum in the $z$ direction, is of order of $M v_{\phi}$, where $v_{\phi}$ is the tangential velocity of the star near the nucleus at an average distance $r=\left\langle r_{0}\right\rangle$. Thus we have

$M \sum_{i=1}^{n} \Delta p_{z i} \approx<F_{z}>\sum_{i=1}^{n} \Delta t_{i}$.

If we set

$M \sum_{i=1}^{n} \Delta p_{z i}=M v_{\phi}=M L_{z c} /<r_{0}>, \sum_{i=1}^{n} \Delta t_{i}=T_{\mathrm{c}}$

and combine Eqs. (5) and (6), we find

$M L_{z c} /<r_{0}>\approx<F_{z}>T_{\mathrm{c}}$.

The force acting in the $z$-direction for a star of unit mass $M=1$ is

$F_{z}=-\frac{\alpha z}{r^{2}+\alpha z^{2}+c^{2}}$.

Setting $z=c, r=\left\langle r_{0}\right\rangle \gg z$ (remember that the star before scattering is very close to the galactic plane) and keeping only the linear terms in $z$ and $c$ Eq. (8) becomes

$<F_{z}>\approx-\frac{\alpha c}{<r_{0}>^{2}}$. 


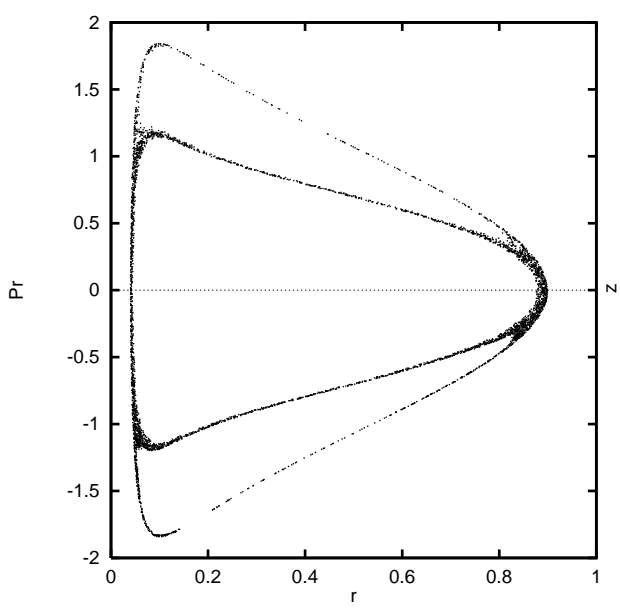

(a)

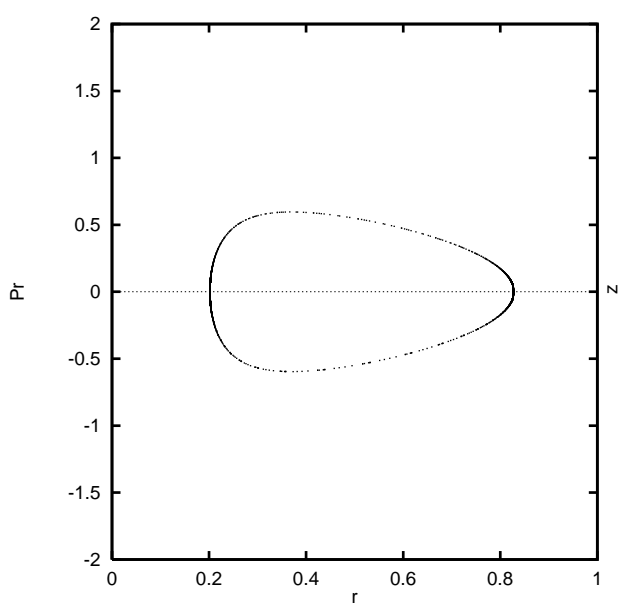

(c)

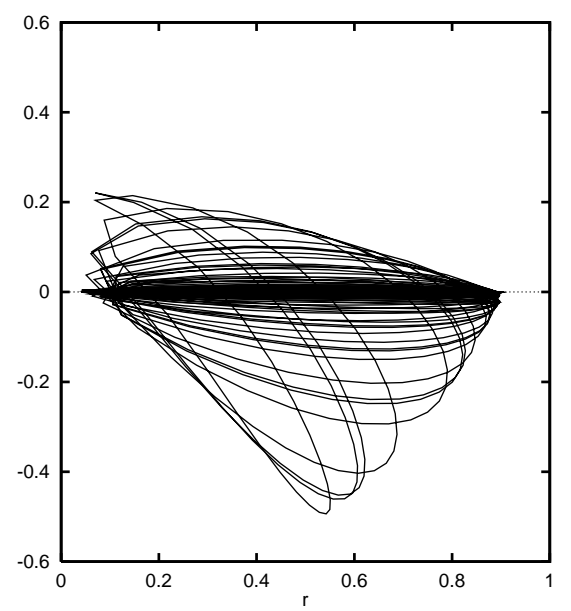

(b)

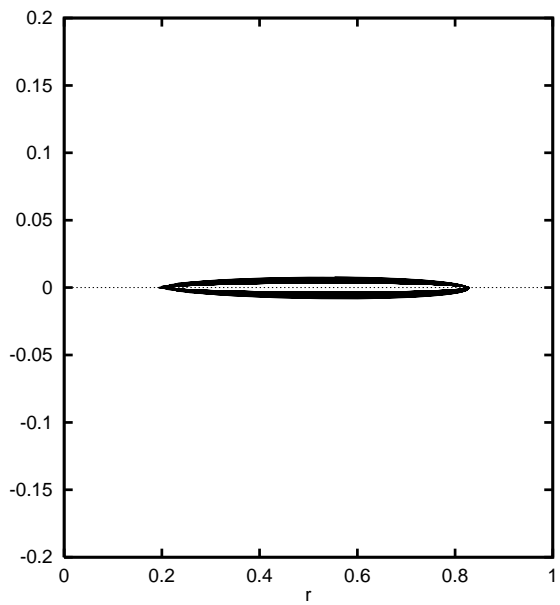

(d)

Fig. 2. a-d) The $r-p_{r}$ phase plane and the corresponding trajectories when $\left.L_{z}=0.10 \mathbf{a}, \mathbf{b}\right)$ and when $\left.L_{z}=0.35 \mathbf{c}, \mathbf{d}\right)$. The values of $h$ and $c$ are -0.1 and 0.01 respectively. Note that regular orbits stay close to the galactic plane while chaotic orbits reach high values of $z$

Inserting the value of $F_{z}$ from Eq. (9) into Eq. (7) we obtain

$<L_{z c}>\approx-\frac{\alpha c}{<r_{0}>} T_{\mathrm{c}} \approx-\alpha k c$

where $k=T_{\mathrm{c}} /<r_{0}>$. Here we must note that Eq. (10) cannot be considered as an exact representation of the relation between the involved quantities. It, rather, can be seen as an indication of the relation that needs to be completed with additional terms. Those terms can be derived through numerical experiments providing the necessary information. Actually, numerical calculations suggest that expression (10) needs to be supplemented with an additional constant term giving the value of $L_{z c}$ in the case $c \rightarrow 0$. Calling this term $L_{z c 0}$ expression (10) takes the form

$<L_{z c}>\approx-\alpha k c+L_{z c 0}$
Equation (11) explains the linear relationship between $c$ and $L_{z c}$ shown in Fig. 1. As we observe the slope of the line in Fig. 1 changes for very small values of $c$. This happens because $\left\langle r_{0}\right\rangle$ becomes smaller in this area while the change in the duration $T_{\mathrm{c}}$ of the scattering is negligible. Equation (11) also contains the flattening parameter $\alpha$. As one can see, for a given value of $c$, there is a linear dependence between $\alpha$ and $L_{z c}$. This linear dependence is also observed in numerical experiments. Figure 3 shows a plot between $\alpha$ and $L_{z c}$ obtained numerically. The value of $c$ is 0.01 while $h=-0.1$.

Our numerical calculations also revealed another interesting phenomenon. The chaotic region decreases as $\alpha$ decreases. For a given value of $L_{z}<L_{z c}$ the chaotic region decreases as $\alpha$ goes from 2 to 1.5. Considerable chaotic zones are observed for $\alpha=2$ while when $\alpha \leq 1.5$ the chaotic regions become negligible. This suggests that flat elliptical galaxies with dense central concentrations 


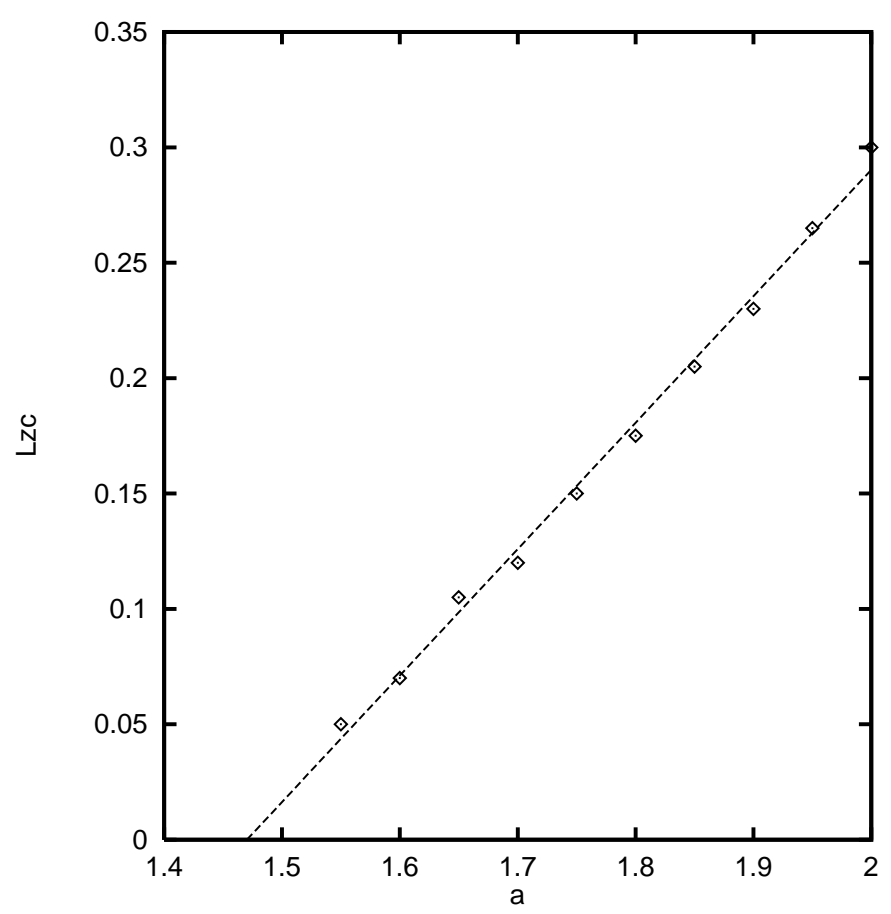

Fig. 3. Relationship between the flattening parameter $\alpha$ and the critical value of the angular momentum $L_{z c}$ when $c=0.01$, $h=-0.1$

display chaotic motion. On the other hand, as we go to spherical systems the chaotic phenomena decrease dramatically. We speak for dense central concentrations because for large radius of the central mass, chaotic phenomena are not observed. Extensive numerical calculations suggest that, when the radius of the bulge is $c \geq 0.15$, the chaotic phenomena are negligible. Note that this behavior is similar to that observed in disk galaxies (see Caranicolas \& Innanen 1991), where we observed chaos only when the scale length of the bulge was smaller or equal to $1.3 \mathrm{kpc}$.

\section{Spectra of orbits}

During the last years astronomers have frequently used the distribution of the values of various parameters along an orbit in order to distinguish between regular and chaotic motion in galactic potentials. One of the well known parameters used for such studies is the stretching number (see Contopoulos et al. 1995; Caranicolas \& Vozikis 1999). The "stretching number" $\alpha_{i}$ is defined as

$\alpha_{i}=\ln \left|\frac{\xi_{i+1}}{\xi_{i}}\right|$,

where $\xi_{i+1}$ is the next image on the Poincare phase plane of an infinitesimal deviation $\xi_{i}$ between two nearby orbits. The spectrum of the stretching numbers is their distribution function

$S(\alpha)=\frac{\Delta N(\alpha)}{N \mathrm{~d} \alpha}$ where $\Delta N(\alpha)$ is the number of stretching numbers in the interval $(\alpha, \alpha+\mathrm{d} \alpha)$ after $N$ iterations.

In the following, instead of using the distribution of stretching numbers, we use the distribution of the radial velocities $v_{r i}$, where $v_{r i}$ are the successive values of the radial velocity on the Poincare $r-p_{r}\left(p_{r}=v_{r}\right)$ surface of section. We shall call the dynamical spectrum of the parameters $v_{r}$ its distribution function

$S\left(v_{r}\right)=\frac{\Delta N\left(v_{r}\right)}{N \mathrm{~d} v_{r}}$,

where $\Delta\left(v_{r}\right)$ are the number of the parameters $v_{r}$ in the interval $\left(v_{r}, v_{r}+\mathrm{d} v_{r}\right)$ after $N$ iterations. The basic reason for using the spectrum $S\left(v_{r}\right)$ is that it is fast and reliable. This happens because we need only one orbit for the calculations and a relatively small number of iterations (about $10^{4}$ ) for reliable information.

Figures $4 \mathrm{a}-\mathrm{d}$ show the $S\left(v_{r}\right)$ spectrum of four orbits, corresponding to four values of the flattening parameter $\alpha$ in the potential (1). All orbits have the same initial conditions $r=0.8992, z=p_{r}=0$ and the same value of $L_{z}=10$. The values of other parameters are $h=-0.1$, $c=0.01$ while the number of iterations for all spectra is $N=10^{4}$. The spectra shown in Figs. $4 \mathrm{a}-\mathrm{c}$ correspond to chaotic orbits while the spectrum shown in Fig. 4d correspond to a regular orbit. The values of the flattening parameter $\alpha$, for the spectra shown in Figs. 4a-c, are 2, 1.75 and 1.6 respectively. The value of $\alpha$ for the last Fig. $4 \mathrm{~d}$ is 1.5. It is interesting to observe that as we go from the value $\alpha=2$ to the value $\alpha=1.5$ the spectrum $S\left(v_{r}\right)$ changes structure and tends from a typical asymmetric chaotic spectrum, with large and small peaks, to a typical "U" type symmetric regular spectrum. In other words the distribution of the radial velocities, on the $r-p_{r}$ Poincare surface of section, for the four orbits corresponding to Figs. $4 \mathrm{a}-\mathrm{d}$ become gradually from random and asymmetric to organized and symmetric. Therefore the evolution of the $S\left(v_{r}\right)$ spectrum as the flattening parameter goes from 2 to 1.5 , shows in a very illustrative way the gradual change of the trajectories from chaotic to regular.

\section{Discussion}

In this paper we have studied the transition from regular to chaotic motion in a logarithmic galactic potential (1). This potential is considered to describe global motion in an elliptical galaxy with a nucleus or bulge of radius $c$. The magnitude of the radius $c$ together with the value of angular momentum $L_{z}$ are two basic parameters for the system to display regular or chaotic motion. It was found numerically that a linear relationship exists between the critical value of the angular momentum and the corresponding radius $c$ of the central concentration. The above mentioned relationship can also be obtained using seminumerical methods. 


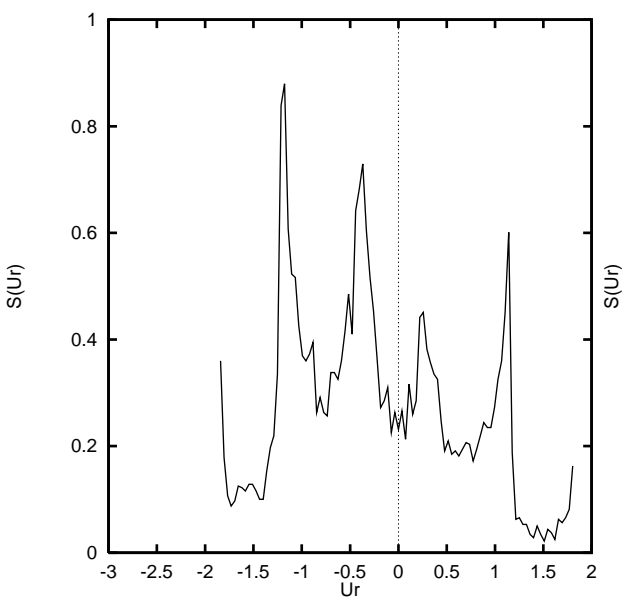

(a)

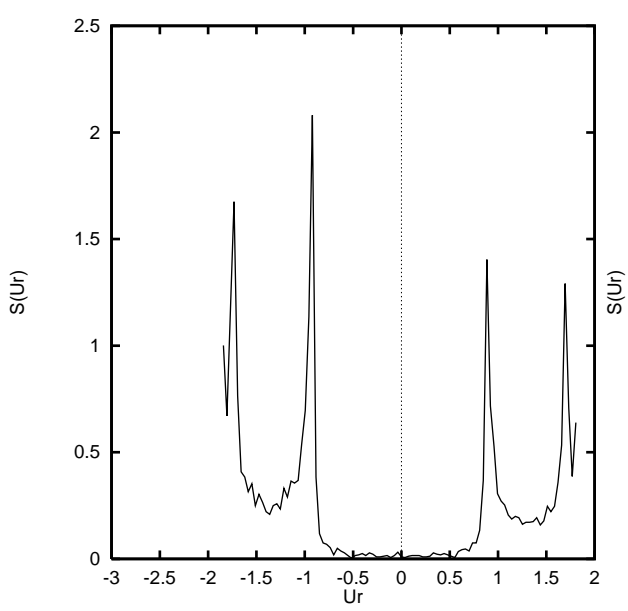

(c)

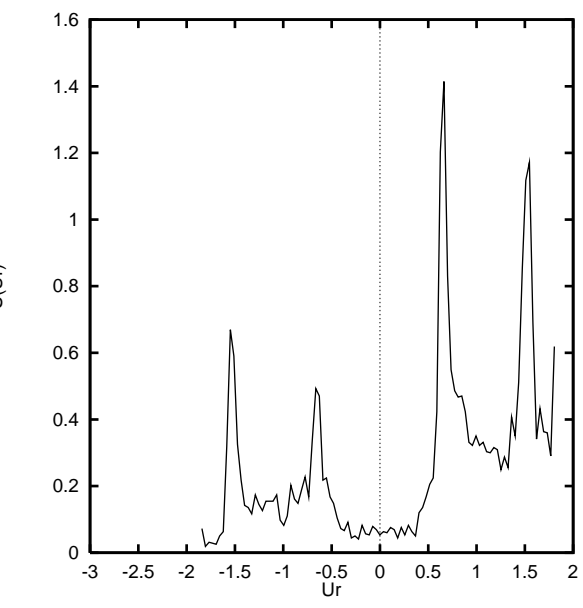

(b)

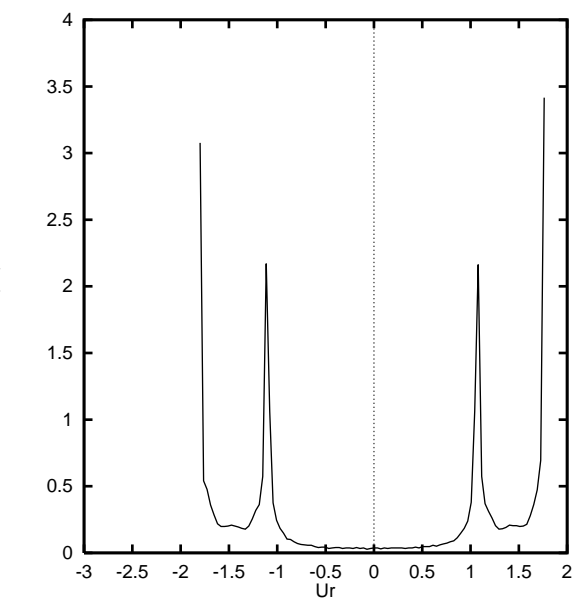

(d)

Fig. 4. a-d) The $S\left(v_{r}\right)$ spectra for the four orbits described in the text. The values of the flattening parameter $\alpha$ in the Figs. a), b), c) and d) are 2.0, 1.75, 1.6 and 1.5 respectively. It is interesting to note the gradual change of the spectrum from chaotic to regular as the flattening parameter $\alpha$ goes from 2 to 1.5

An important role on the evolution of the chaotic motion is played by the flattening parameter $\alpha$ of the system. For a given value of the radius $c$, there is a linear relationship between $\alpha$ and the critical value of the angular momentum $L_{z c}$. We must emphasize that the chaotic phenomena are negligible when $\alpha \leq 1.5$ and when $c \geq 0.15$. This strongly suggests that, low angular momentum stars, display chaotic motion in highly flattened elliptical galaxies having a dense nucleus or bulge. Here we must note that this behavior is similar to that observed in disk galaxy models studied by Caranicolas \& Innanen (1991).

In order to visualize the evolution of the chaotic phenomena, as the flattening parameter $\alpha$ changes, we used the $S\left(v_{r}\right)$ spectrum. We introduced and used this kind of spectrum, which is based on the distribution of values of the radial velocity on the $r-p_{r}$ phase plane, because it describes in a very illustrative way the transition from regular to chaotic motion, as of the system tends to be "more spherically symmetric" by decreasing $\alpha$ from 2 to 1.5. The advantage of this spectrum is that it is fast because it needs only one orbit in order to find the distribution of values of the radial velocity on the $r-p_{r}$ phase plane and not two nearby trajectories as the spectrum of the stretching numbers does. The gradual change of the orbital behavior of the system (1), from chaotic to regular, as the flattening parameter decreases, is reflected on the $S\left(v_{r}\right)$ spectrum which undergoes a significant change from a typical chaotic spectrum, when $\alpha=2$, to the regular "U" type spectrum for $\alpha=1.5$.

Finally we must note that the equipotential curves, in the $(r, z)$ plane, for the system (1) are only a third as flattened as the contours of equal density and the density becomes negative on the $z$-axis when $\alpha>2$ (see Binney \& Tremaine 1987, p. 48).

Acknowledgements. Our thanks go to an anonymous referee for his useful suggestions and comments. 


\section{References}

Binney, J., \& Tremaine, S. 1987, Galactic Dynamics (Princeton University press, Princeton, New Jersey)

Caranicolas, N. D. 1990, Cel. Mec., 47, 87

Caranicolas, N. D. 1997, A\&SS, 246, 15

Caranicolas, N. D., \& Innanen, K. A. 1991, AJ, 103, 1308

Caranicolas, N. D., \& Karanis, G. I. 1999, A\&A, 342, 389

Caranicolas, N. D., \& Vozikis, Ch. 1986, Cel. Mech., 39, 105

Caranicolas, N. D., \& Vozikis, Ch. 1999, A\&A, 449, 70

Contopoulos, G., \& Seimenis, J. 1990, A\&A, 227, 49
Contopoulos, G., Grousousakou, E., \& Voglis, N. 1995, A\&A, 304,374

Henrard, J. 1988, Resonances in the planar Elliptic Restricted Problem, In Long Term behavior of Natural and Artificial $N$-body Systems, ed. A. E. Roy

Henrard, J., \& Caranicolas, N. D. 1990, Cel. Mech., 47, 99 Innanen, K. A. 1985, AJ, 90, 2377

Lemaitre, A. 1984, Cel. Mech., 32, 109

Papaphilippou, Y., \& Laskar, J. 1996, A\&A, 307, 427

Richstone, D. 1980, ApJ, 238, 103

Richstone, D. 1982, ApJ, 252, 496 